\title{
Comprehensive mapping infection-enhancing epitopes of dengue pr protein using polyclonal antibody against prM
}

\author{
Yayan Luo ${ }^{1} \cdot$ Xiaolan Guo ${ }^{2} \cdot$ Huijun Yan $^{2} \cdot$ Danyun Fang ${ }^{2} \cdot$ Gucheng Zeng ${ }^{2} \cdot$ \\ Junmei Zhou ${ }^{2} \cdot$ Lifang Jiang ${ }^{2}$
}

Received: 4 February 2015 /Revised: 11 March 2015 / Accepted: 12 March 2015 /Published online: 31 March 2015

(C) The Author(s) 2015. This article is published with open access at Springerlink.com

\begin{abstract}
Dengue vaccine development is considered a global public health priority, but the antibody-dependent enhancement (ADE) issues have critically restricted vaccine development. Recent findings have demonstrated that pre-membrane (prM) protein was involved in dengue virus (DENV) infection enhancement. Although the importance of prM antibodies have been well characterized, only a few epitopes in DENV prM protein have ever been identified. In this study, we screened five potential linear epitopes located at positions pr1 (1-16aa), pr3 (13-28aa), pr4 (19-34aa), pr9 (49-64aa), and pr10 (55-70aa) in pr protein using peptide scanning and comprehensive bioinformatics analysis. Then, we found that
\end{abstract}

Junmei Zhou

junmeizh@126.com

Lifang Jiang

jianglf@mail.sysu.edu.cn

Yayan Luo

yayanluo@163.com

Xiaolan Guo

xiaolan-g@126.com

Huijun Yan

hjyan@126.com

Danyun Fang

danyunf@126.com

Gucheng Zeng

zenggch@mail.sysu.edu.cn

1 Guangzhou Brain Hospital (Guangzhou Huiai hospital, the affiliated hospital of Guangzhou Medical University), Guanghzou 510370, China

2 Key Laboratory for Tropic Diseases Control, Ministry of Education of China, Department of Microbiology, Zhongshan School of Medicine, Sun Yat-sen University, Guangzhou 510080, China only pr4 (19-34aa) could elicit high-titer antibodies in Balb/c mice, and this epitope could react with sera from DENV2infected patients, suggesting that specific antibodies against epitope peptide pr4 were elicited in both DENV-infected mice and human. In addition, our data demonstrated that anti-pr4 sera showed limited neutralizing activity but significant ADE activity toward standard DENV serotypes and imDENV. Hence, it seems responsible to hypothesize that anti-pr4 serum was infection-enhancing antibody and pr4 was infectionenhancing epitope. In conclusion, we characterized a novel infection-enhancing epitope on dengue pr protein, a finding that may provide new insight into the pathogenesis of DENV infection and contribute to dengue vaccine design.

Keywords Dengue virus $\cdot$ pr protein $\cdot$ Epitope $\cdot$ Synthetic peptides $\cdot$ Antibody-dependent enhancement

\section{Introduction}

Dengue virus (DENV1-4) causes the most significant arboviral disease in the tropical and subtropical areas of the world, with an estimated 390 million infections per year (Guzman and Kouri 2002). While most DENV infections are asymptomatic or lead to self-limited dengue fever (DF), a growing number of patients present potentially fatal clinical manifestations including dengue hemorrhagic fever (DHF) and dengue shock syndrome (DSS) (Bhatt et al. 2013). Although infection with DENV is a growing global public health concern and imposes one of the largest socioeconomic burdens in the world, approved vaccine remains unavailable despite decades of effort (Coller and Clements 2011; Miller 2010).

The immunopathogenesis of DENV infection is still poorly understood. It is well established that the development of 
DHF/DSS is associated with sequential infection with different serotypes (Gubler 2006). Also, primary infection of infants born to dengue-immune mothers may be at greater risk of developing severe disease (Simmons et al. 2007). The hypothesis of antibody-dependent enhancement (ADE) of infection has been postulated because of these observations. It is reasoned that subneutralizing antibodies promoted virus uptake into $\mathrm{Fc}$ receptor-bearing cells, leading to increased DENVinfected cells and eventually a higher virus load (Halstead 1970; Halstead and O'Rourke 1977; Halstead 2003). Hence, a successful and safe vaccine must be tetravalent, capable of providing long-term protection against all four serotypes simultaneously (Guy et al. 2011).

Dengue is a single-stranded, positive-sense, RNA virus with a genome of approximately $11 \mathrm{~kb}$. The virion contains three structural (capsid (C), pre-membrane (prM), and envelope (E)) and seven nonstructural (NS) proteins (NS1, NS2a, NS2b, NS3, NS4a, NS4b, and NS5). prM is a 166-amino-acid protein and acts as a chaperone for correct folding of the $\mathrm{E}$ protein during virus assembly and maturation ( $\mathrm{Li}$ et al. 2008; Mukhopadhyay et al. 2005). prM contains a furin cleavage site and is cleaved by the host cell endoprotease furin into a C-terminal M protein and an N-terminal 91-amino-acid precursor peptide ( $\mathrm{pr}$ protein), resulting in the formation of mature infectious virus (Yu et al. 2008). Interestingly, cells infected with DENV secrete a heterogeneous mixture varying from fully mature (containing M), partially mature virions (containing a mixture of prM and $\mathrm{M}$ ) to fully immature (containing prM) due to inefficient cleavage of prM to $\mathrm{M}$ by furin during DENV maturation (Rodenhuis-Zybert et al. 2008; Cherrier et al. 2009; Junjhon et al. 2010). It has been proved that fully immature virus particles are inherently noninfectious whereas fully mature and partially mature virus are infectious (Rodenhuis-Zybert et al. 2008, 2010, 2011; Dejnirattisai et al. 2010). Additionally, the enhancement or neutralization effect of antibodies seems to be dependent on the maturation state of virions (Dejnirattisai et al. 2010; Rodenhuis-Zybert et al. 2010; Nelson et al. 2008).

It has been previously reported that anti-prM antibodies are commonly found in sera of patients with DENV infection (Dejnirattisai et al. 2010; Bray and Lai 1991; Cardosa et al. 2002; Se-Thoe et al. 1999). However, the potential role of prM antibodies during DENV infection was long overlooked as numerous functional studies have revealed that fully immature particles are noninfectious (Colpitts et al. 2011). Interestingly, recent studies on mouse and human antibodies showed that prM antibodies render virtually noninfectious immature DENV particles highly infectious (Dejnirattisai et al. 2010; Rodenhuis-Zybert et al. 2010; Chan et al. 2012). In addition, anti-prM antibodies are found to play important roles in patients with both primary infection and second infection (Dejnirattisai et al. 2010; Lai et al. 2008). Furthermore, many studies have suggested that these antibodies against prM do not well neutralize DENV infection but potently promote ADE infection among four DENV serotypes (Dejnirattisai et al. 2010; Beltramello et al. 2010). It has also been suggested that anti-prM antibodies could enhance wild-type DENV infection (Huang et al. 2005, 2006) and increase disease severity in infants upon primary infection (Chau et al. 2009). Most importantly, previous study showed a positive correlation between the circulating prM antibody level and disease severity (Rai et al. 2008). Taken together, these studies suggest that prM-specific monoclonal antibodies (mAbs) have a potential role to enhance DENV infection in humans and thus may contribute to the development of severe disease.

A recent report detecting the acute $\mathrm{B}$ cell response in dengue patients has found that DENV infection results in significant B cell activation at days 4-7 after onset of fever (Balakrishnan et al. 2011). prM protein, like $\mathrm{E}$ protein, is a major target in the humoral immune response to DENV (Cardosa et al. 2002; Beltramello et al. 2010). Epitopes are the focus of pathogenesis research as well as the development of vaccine and diagnostic reagent (Wu et al. 2001, 2003; Jiang et al. 2010). Epitopes in the $\mathrm{E}$ ( $\mathrm{Li}$ et al. 2012; de Alwis et al. 2012; Lin et al. 2012; Hughes et al. 2012; Wahala et al. 2012), NS1( Wu et al. 2001, 2003; Jiang et al. 2010; Steidel et al. 2012), NS4a, and C (Anandarao et al. 2005) have been well mapped. Although there have been attempts to locate the epitopes of prM (Falconar 1999; Huang et al. 2008; Song et al. 2013; Luo et al. 2013), the functional roles of the prM protein in DENV infection and the precise antigenic structures of prM are not yet fully understood. Thus, in this study, we mapped the potential B cell epitopes in the pr protein of DENV with peptide scanning and comprehensive bioinformatics analysis. We also investigated the neutralizing versus enhancing capacity of antisera of epitope peptide pr4 toward standard DENV14 particles and imDENV particles.

\section{Materials and methods}

\section{Cells and viruses}

C6/36 cells, baby hamster kidney-21 (BHK-21) cells, human adenocarcinoma LoVo cells, and human erythroleukemic K562 cells were maintained as previously described (Luo et al. 2013). All cells were purchased from ATCC.

DENV1 strain Hawaii (GenBank EU848545), DENV2 strain New Guinea C (NGC) (GenBank AF038403), DENV3 strain H87 (GenBank M93130), DENV4 strain H241 (GenBank AY947539), and JEV (GenBank AF315119) were propagated on $\mathrm{C6} / 36$ cells, while fully imDEVN2 NGC strain was produced on furin-deficient LoVo cells as described before (Rodenhuis-Zybert et al. 2008; Luo et al. 2013). The infectious titers of virus particles were detected by plaque assay on BHK-21 cells and viral RNA levels were measured 
by real-time quantitative RT-PCR (qRT-PCR) (Luo et al. 2012). All viruses were kindly provided by Chinese Center for Disease Control and Prevention, Beijing, China. All viruses were deposited in GenBank.

\section{Antibodies and human serum samples}

2H2 (IgG2a anti-DENV1-4 prM) and 4G2 (IgG2a anti-all flavivirus E) hybridomas were purchased from ATCC.

To generate polyclonal antibody (pAb) against prM, the DENV-2 prM protein was expressed in E. coli and then purified by electroelution. The purified protein was used to immunize adult male New Zealand rabbits to produce $\mathrm{pAb}$ directed to prM. Anti-prM pAb was purified by caprylic acid-ammonium sulfate precipitation and protein A affinity column (GenScript, USA); then, the specificity and titer of the antibody were verified by Western blot and enzyme-linked immunosorbent assay (ELISA) (Feng et al. 2013).

Human serum samples were obtained and identified as described before (Luo et al. 2013). The use of human sera was approved by the ethical committee of Haizhu District Center for Disease Control and Prevention of Guangzhou, China. The study was also approved by the Ethics Committee of Sun Yatsen University.

\section{Peptide synthesis and epitope mapping using anti-prM pAb}

Fourteen overlapping peptides (each with 16 amino acids, overlap of 10) covering the pr protein were designed and synthesized (purity $>95 \%$, China Peptides Co., Ltd) (Table 1). Then, the predominant linear B epitopes on pr protein were mapped by ELISA using anti-prM pAb and 14 overlapping peptides.

\section{ELISA}

To screen specific epitopes that directly bind with the pr protein, ELISA was performed with anti-prM pAb. The ELISA plate was coated with $100 \mu \mathrm{l}$ synthetic peptides at a concentration of $10 \mu \mathrm{g} / \mathrm{ml}$ at $4{ }^{\circ} \mathrm{C}$ overnight and blocked at $37^{\circ} \mathrm{C}$ for $2 \mathrm{~h}$. After washing three times with PBS with $0.1 \%$ Tween-20
(PBST), the plates were incubated with $100 \mu \mathrm{l}$ anti-prM pAb diluted in 1:200 at $37{ }^{\circ} \mathrm{C}$ for $1 \mathrm{~h}$. Bound antibodies were detected with 1:5000 diluted HRP-conjugated goat-antirabbit $\operatorname{IgG}$ at $37{ }^{\circ} \mathrm{C}$ for $45 \mathrm{~min}$. Then, plates were washed three times and incubated with TMB substrate. The reaction was stopped with $2 \mathrm{~N} \mathrm{H}_{2} \mathrm{SO}$, and the plates were measured at $450 \mathrm{~nm}$ using a microplate autoreader.

\section{Bioinformatics analysis of DENV2 pr B cell epitopes}

The potential linear epitope on pr protein was predicted using DNASTAR software and ExPaSy multiple bioinformation software as described previously (Luo et al. 2013).

\section{Competitive-inhibition assay}

The ELISA plate was coated with anti-prM pAb. Next, synthetic peptide was added $0.1 \mu \mathrm{g}$ per well and purified prM protein was added simultaneously. Then, the same procedures as described in "ELISA" were followed.

\section{Immunization assay and protection assay in adult $\mathrm{Balb} / \mathrm{c}$ mice}

All procedures involving animals were approved by the Animal Experimentation Ethics Committee of Sun Yat-sen University and carried out by a licensed individual with an ethical approval number of 2012/0081.

Considering the results of peptide scanning and bioinformatics analysis together, we screened five predominant linear B epitopes located at regions pr1 (1-16aa), pr3 (13-28aa), pr4 (19-34aa), pr9 (49-64aa), and pr10 (55-70aa). Then, we chose epitope peptides (pr1, pr3, pr4, pr9, and pr10) and control peptide PM10 (SQNPPHRHQS) to immunize Balb/C mice and collected anisera for further study as described previously (Luo et al. 2013). The antibody titer of mice sera was detected by using ELISA.

The protection experiment was performed as described previously (Luo et al. 2013). The viral RNA levels of serum samples were detected using qRT-PCR (Luo et al. 2012). All animals were euthanized by using carbon dioxide $\left(\mathrm{CO}_{2}\right)$
Table 1 A total of 14 overlapping peptides of pr protein (each with 16 amino acids, overlap of 10)

\begin{tabular}{|c|c|c|c|}
\hline Peptide & Peptide sequence and location & Peptide & Peptide sequence and location \\
\hline pr1 & ${ }^{1}$ fhlttrngephmivsr ${ }^{16}$ & pr8 & ${ }^{43}$ elcedtityncpllrq ${ }^{58}$ \\
\hline pr2 & ${ }^{7}$ ngephmivsrqekgks ${ }^{22}$ & pr9 & ${ }^{49}$ ityncpllrqnepedi $^{64}$ \\
\hline pr3 & ${ }^{13}$ ivsrqekgksllfkte ${ }^{28}$ & pr10 & ${ }^{55} 1$ lrqnepedidcwcns ${ }^{70}$ \\
\hline pr4 & ${ }^{19}$ kgksllfktengvnme $^{34}$ & pr11 & ${ }^{61}$ pedidcwenststwvt ${ }^{76}$ \\
\hline pr5 & ${ }^{25}$ fktengvnmctlmamd ${ }^{40}$ & $\operatorname{pr} 12$ & ${ }_{67} \mathrm{Wcnststwvtygtcta}^{82}$ \\
\hline pr6 & ${ }^{31}$ vnmctlmamdlgelce ${ }^{46}$ & pr13 & ${ }^{73}$ twvtygtctatgehrr ${ }^{88}$ \\
\hline pr7 & ${ }^{37}$ mamdlgelcedtityn ${ }^{52}$ & pr14 & ${ }^{79}$ tctatgehrrekr $^{91}$ \\
\hline
\end{tabular}


according to NC3Rs standard procedures, and the experiment was terminated.

\section{Plaque reduction neutralization test (PRNT) and antibody-dependent infection enhancement (ADE)} assay

To determine the ability of prM-specific antibodies to neutralize or enhance DENV infection in vitro, PRNT and ADE assay were carried out on BHK-21 cells and K562 cells respectively as described in our previous study (Luo et al. 2013).

\section{Flow cytometry}

The infected K562 cells were fixed, permeabilized, and stained with 4G2 conjugated to Alexa-Fluor-488 (Invitrogen). The percent of infected cells was determined by flow cytometry.

\section{Statistical analysis}

Statistical analyses were performed in GraphPad Prism5.0 software. ANOVA Tukey's posthoc statistical tests were used for pairwise comparisons of multiple groups. A $p$ value of less than 0.05 was considered statistically significant.
Fig. 2 General evaluation of DENV2-pr epitopes with bioinformation software. a Hopp and Wood hydrophilicity, b Granthan polarity, c Bhaskaran and Ponnuswamy flexibility, d Deleage and Roux alphahelix regions, e Deleage and Roux beta-turn regions, f Jameson and Wolf antigenicity, and $\mathbf{g}$ Emini accessibility

\section{Results}

Mapping of the antigenic epitopes on the pr protein using anti-prM pAb

To identify the potential antigenic epitopes on the pr protein, 11 partially overlapping peptides (pr1-pr14) were screened by ELISA using anti-prM pAb at various dilutions. The results showed that seven peptides (pr1, pr3, pr4, pr6, pr9, pr10, and pr11) were recognized by anti-prM pAb (Fig. 1), suggesting that these peptides may be the potential B cell epitopes in pr protein. All the other seven overlapping peptides and control peptide PM10 failed to bind with pAb against prM. Our previous study (Luo et al. 2013) has reported that the potential linear epitopes on pr protein predicted using DNAS TAR software and ExPaSy multiple bioinformation software were located at regions 3-12aa, 12-26aa, 55-66aa, and 82-91aa (Fig. 2). Considering the results of peptide scanning and bioinformatics analysis together, five predominant epitope peptides ( $\mathrm{pr} 1, \mathrm{pr} 3, \mathrm{pr} 4, \mathrm{pr}$, and pr10) were chosen to immunize Balb/C mice for further study.
Fig. 1 Comprehensive identification of linear B cell epitopes in DENV2 pr protein using anti-prM pAb by ELISA. Seven potential B cell epitopes in pr protein were mapped. prM pAb was diluted 200-fold (a), 1000fold (b), 5000-fold (c), and 10,000-fold (d). PM10 was used control peptide. Data are expressed as means of at least three independent experiments. The error bars represent standard deviations (SD). If there is no error bar, it is not that no variations among three independent experiments but that the variations are too small to show in the figure
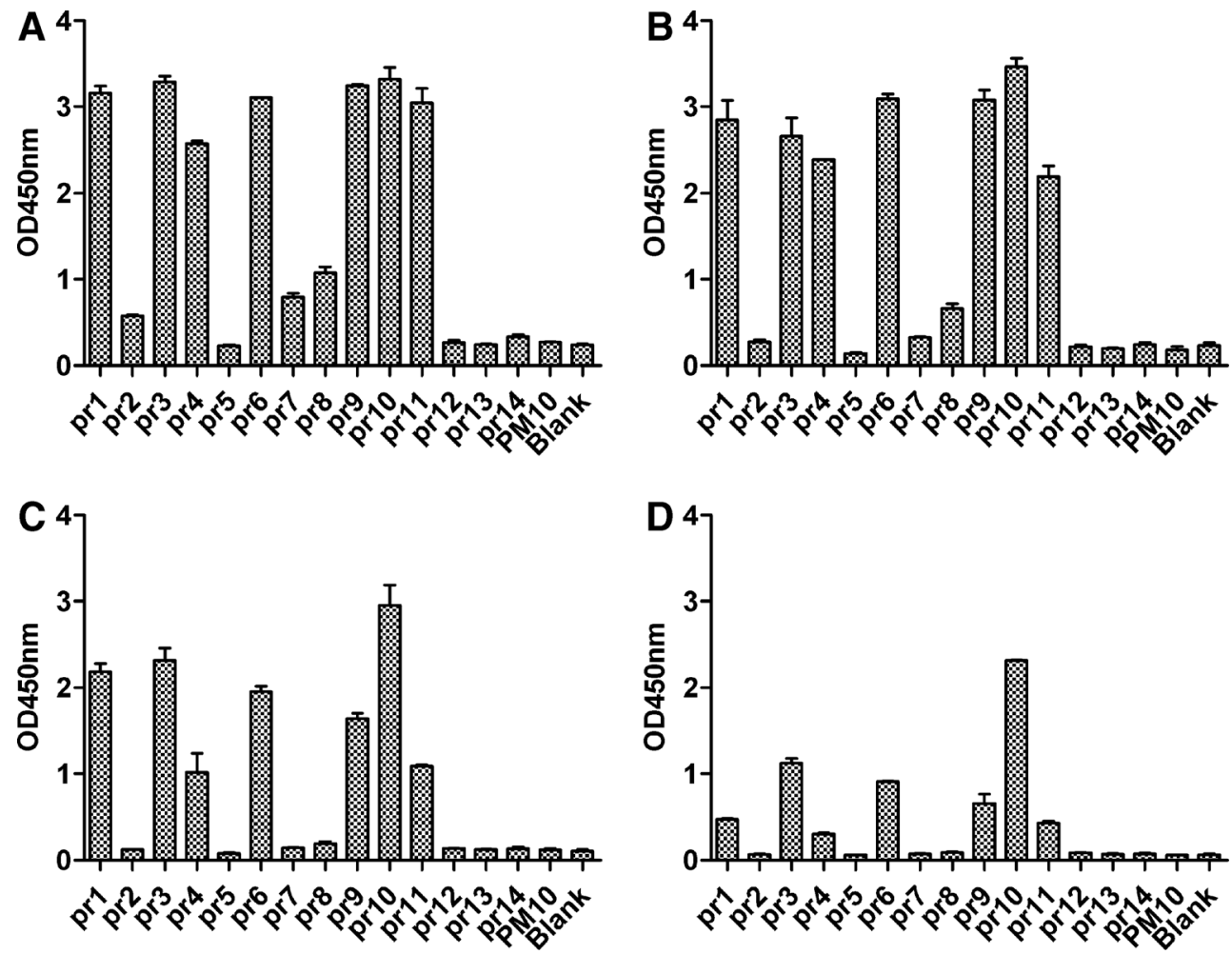

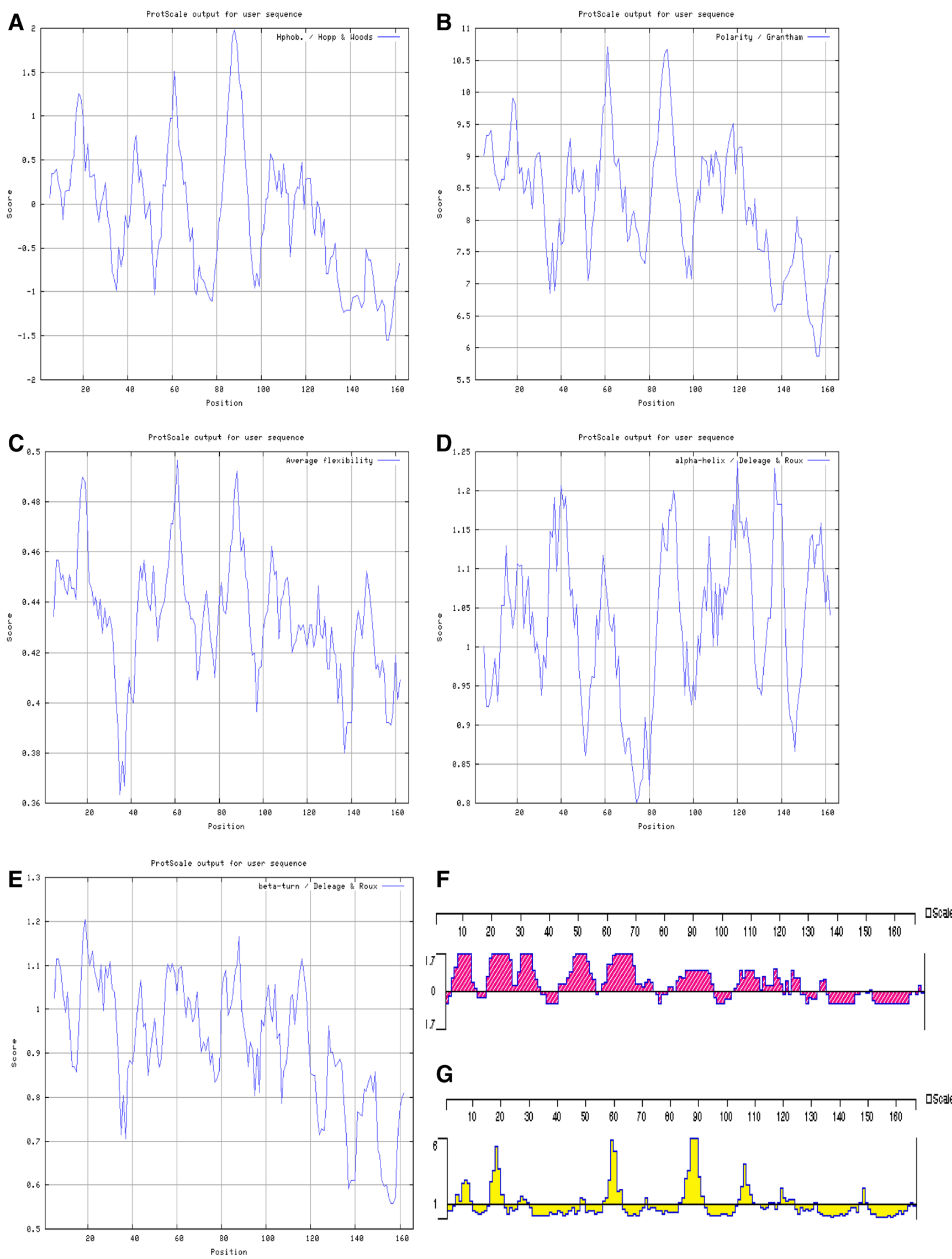

G
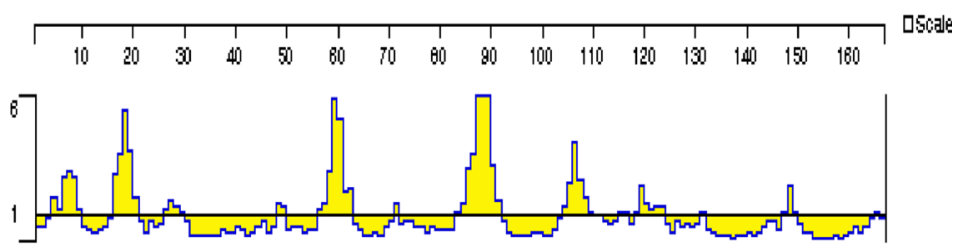
Immunogenicity of synthetic peptides on Balb/c mice

According to ELISA results, high titer of antibodies was only found in mice immunized with pr4. The antibody titer of antipr4 sera was remarkably higher than that of the antisera from groups of pr1, pr3, pr9, pr10, PM10, and PBS at any time point $(p<0.05)$. Furthermore, antibody titers of pr4 increased progressively with each sequential immunization. Thus, immunization with pr4 elicits high-titer antibodies in Balb/c mice (Fig. 3).

\section{Protection response in adult Balb/c mice}

As shown in Fig. 4, high levels of viral RNA in all groups were observed at days 0.25 and 1 post-infection, and the viral RNA levels in the pr4 group were significantly higher than that in the other groups (pr1, pr3, pr6, pr9, PM10, and PBS) at days 0.25 and 1 post-infection $(p<0.05)$. However, all groups showed a rapidly decreased viremia levels at days $2,3,4$, and 5 post-infection.

\section{Characterization of epitope peptide pr4}

To analyze the conservation of epitope peptide pr4 (19-34aa), we performed a sequence alignment of the pr protein sequences of DENV1-4, YFV, WNV, JEV, and TBEV using DNASTAR software. Alignment results showed that pr4 epitope sequence was highly conserved among four DENV serotypes but not among other flaviviruses (Table 2).

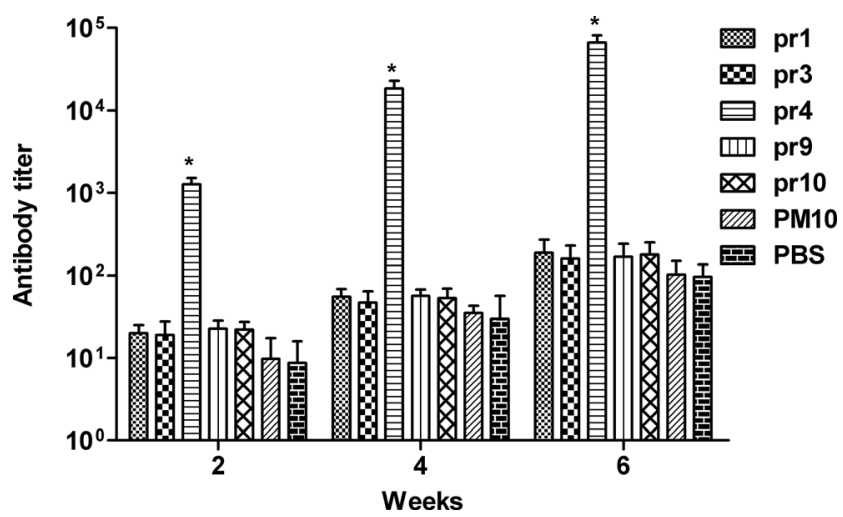

Fig. 3 Serum antibody titers induced in Balb/C mice immunized with the synthetic peptides. Seven groups (pr1, pr3, pr4, pr9, pr10, PM10, and PBS), ten mice for every group, were immunized three times with $100 \mu \mathrm{g}$ of immunogens. All peptides were coupled to KLH before immunization. PM10 and PBS were used as controls. The antisera were collected on week 2, 4 and 6 via tail vein, and the antibody titer of mice sera was detected by ELISA. The antibody titer of anti-pr 4 sera was remarkably higher than that of the antisera from groups of pr1, pr3, pr9, pr10, PM10, and PBS at any time point. Data are expressed as means of at least three independent experiments. The error bars represent standard deviations (SD); ${ }^{*} p<0.05$ vs other groups (pr1, pr3, pr9, pr10, PM10, and PBS)
We next evaluated whether the synthetic peptide pr4 could be recognized by anti-DENV1-4 mice sera. Synthetic peptide pr4 could react with anti-DENV1-4 mice sera but not with anti-JEV mice sera and normal mice sera (NMS) (Fig. 5a), suggesting that synthetic peptide pr4 is a DENV serocomplex cross-reactive epitope-based peptide.

To prove further that synthetic peptide pr4 was the epitope of pr protein, a peptide competitive inhibition assay was performed to determine whether the pr4 peptide competed with prM protein for reactivity with anti-prM pAb. The reaction activity of anti-prM pAb with prM protein was inhibited markedly by pr4 in a concentration-dependent manner (Fig. 5b).

Then, we confirm the reactivity of synthetic peptide pr4 with 20 DENV2 patient serum samples. As shown in Fig. $5 \mathrm{c}, \mathrm{d}, \mathrm{pr} 4$ was able to react with sera from 18 patients infected with DENV2. The sensitivity of pr4 serologic test was $90 \%$. In addition, patients showed difference in the level of antibody. However, all of the sera from 20 healthy adults failed to bind with pr4. These results demonstrated that specific antibody response against pr4 epitope was elicited during natural DENV infection.

\section{Neutralizing activities of anti-pr4 sera}

Plaque reduction neutralization test results showed that anti-pr4 sera failed to completely neutralize infection with the neutralization level varying from 33 to $60 \%$, and the partial neutralization was cross-reactive among four DENV serotypes (Fig. 6). Anti-pr4 sera, like many other prM antibodies, did not exhibited high neutralizing activity. Also, virtually noninfectious imDENV was neutralized by anti-pr4 sera and the titration curve for standard DENV2 and imDENV2 were similar (Fig. 6). These results suggest that anti-pr4 sera exhibited weak neutralizing activities against standard DENV-4 and imDENV2.

\section{ADE activities of anti-pr4 sera}

The flow cytometry results indicated that anti-pr4 sera was able to enhance infection varying from 6.3 to $87.6 \%$ over a wide range of antibody concentration among four DENV serotypes (Fig. 7a). Then, we detected enhancement of imDENV2 infection using a constant amount of virus particles identical to standard DENV2. We found that infection of virtually noninfectious imDENV was also significantly enhanced by anti-pr4 sera. To further confirm the enhancing capacity of anti-pr4 sera, we evaluated viral RNA copies in infected supernatants using qRT-PCR (Fig. 7b). In agreement with flow cytometry results, anti-pr4 sera caused a significant increase of viral load over a large antibody 


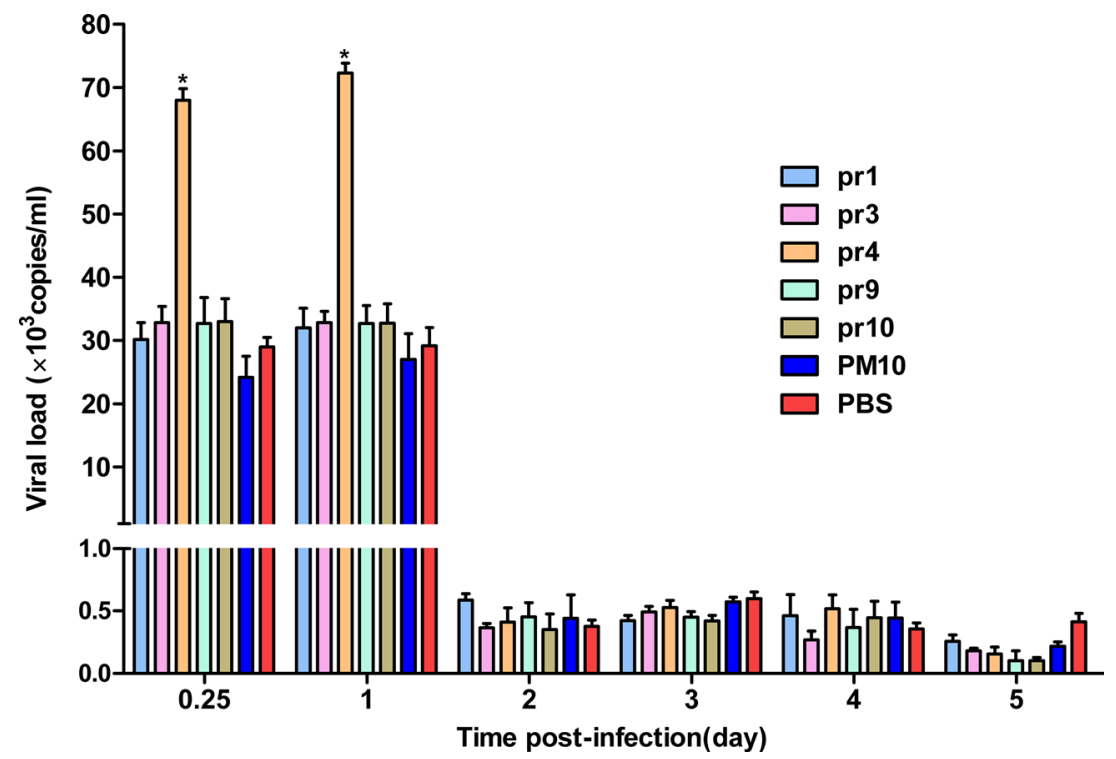

Fig. 4 Viral RNA copy numbers in immunized mice after infected with DENV2 NGC strain. Two weeks after the last immunization, mice were inoculated intraperitoneally with $10^{6}$ PFU DENV2. Viral RNA copy numbers of sera were measured at days $0.25,1,2,3,4$, and 5 postinfection by qRT-PCR. PM10 and PBS were used as controls. The viral

concentration range $(p<0.05)$. In summary, these results suggest that anti-pr4 sera showed significant ADE activities toward standard DENV and imDENV2.

\section{Discussion}

Recent findings demonstrated that anti-prM antibodies play critical roles in human immune responses to DENV. These antibodies showed limited neutralizing activity but significant ADE activity (Dejnirattisai et al. 2010; Rodenhuis-Zybert et al. 2010, 2011; Chan et al. 2012; Lai et al. 2008; Beltramello et al. 2010; Huang et al. 2006). Although the importance of

Table 2 Sequence alignment of amino acid residues 19 to 34 of the pr proteins of flaviviruses

\begin{tabular}{ll}
\hline Virus $^{\mathrm{a}}$ & Amino acid sequence \\
\hline DENV1 & RGKSLLFKTAAGVNMC \\
DENV2 & KGKSLLFKTEDGVNMC \\
DENV3 & RGKSLLFKTASGINMC \\
DENV4 & RGRPLLFKTTEGINKC \\
WNV & VTDVITIPTAAGKNLC \\
JEV & IADVIVIPTSKGENRC \\
YFV & LGKTFSVGTGNCTTNI \\
TBEV & TQVRVENGTCVILATD
\end{tabular}

${ }^{a}$ The protein sequences of DENV1, DENV2, DENV3, DENV4, WNV, JEV, YFV, and TBEV were retrieved from GenBank with accession numbers EU848545, AF038403, M93130, AY947539, DQ211652, AF315119, X03700, and AY182009, respectively
RNA copy numbers in the pr 4 group were significantly higher than that in the other groups (pr1, pr3, pr6, pr9, PM10, and PBS) at days 0.25 and 1 post-infection. Data are expressed as means of at least three independent experiments. The error bars represent standard deviations (SD); ${ }^{*} p<0.05$ vs other groups (pr1, pr3, pr9, pr10, PM10, and PBS)

prM antibodies has been well reported, the precise antigenic structures and the functional roles of the prM protein remain poorly understood. Thus, in this study, we identified the potential B cell epitopes in the dengue pr protein and investigated the ability of antibody against epitope peptide pr4 to enhance infection of standard DENV1-4 and imDENV2.

In the present study, by using peptide scanning and bioinformatics analysis, we mapped five potential linear B cell epitopes (pr1, pr3, pr4, pr9, and pr10) in the dengue pr protein. However, immunization assay results showed that only epitope peptide pr4 was capable of inducing strong humoral immune response in mice and showed to be highly immunogenic. Sequence alignment results demonstrated that $\mathrm{pr} 4$ is a DENV serocomplex cross-reactive epitope peptide. In our experiments, epitope peptide pr4 showed strong reactivity with mouse antisera against DENV1-4 but not with anti-JEV mice sera. Previous studies have reported that anti-prM antibodies could distinguish infection with DENV from infection with JEV (Cardosa et al. 2002; Hua et al. 2010). To our knowledge, cross-reactivity among flaviviruses has been a great diagnostic obstacle, especially for members of DENV. Hence, epitope peptide pr4 could be potentially applied in serological monitoring and differential diagnosis of DENV infection. Most, importantly, human sera from patients infected with DENV2 also specifically recognized pr4, suggesting that epitope peptide pr4 may be valuable for the further development of a DENV-specific diagnostic reagent. Together, these results showed specific antibodies against epitope peptide pr4 were elicited in both DENV-infected mice and human, indicating the importance of pr4 epitope during natural DENV infection. 

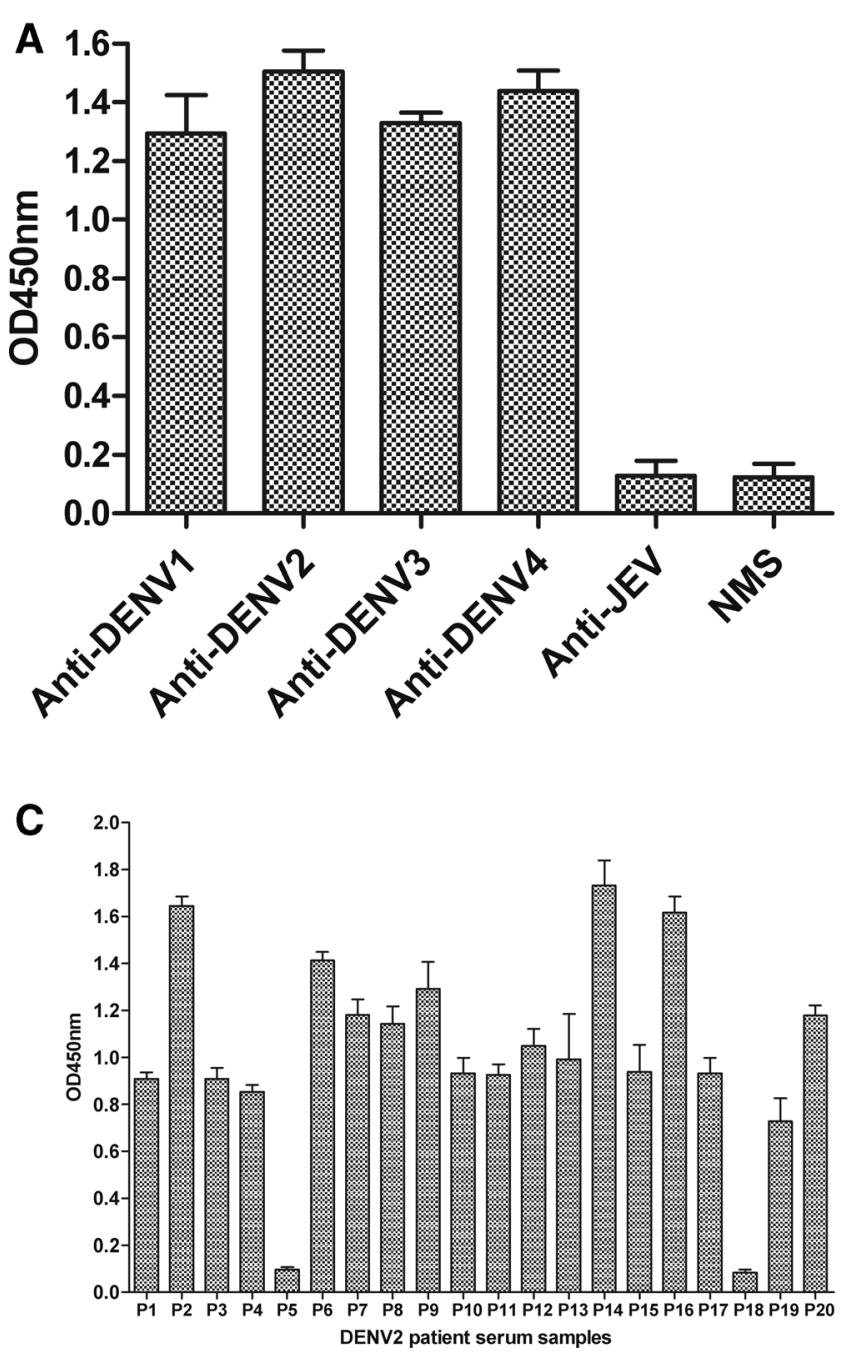

Fig. 5 Characterization of epitope peptide pr4. a ELISA reactivities of synthetic peptide pr4 with immunized mice sera. Synthetic peptide pr4 could react with anti-DENV1-4 mice sera but not with anti-JEV mice sera and normal mice sera (NMS). b Competitive inhibition of prM protein binding to anti-prM pAb by synthetic peptide pr4. Competitive ELISA was performed using pr 4 as competitor of prM protein. The reaction activity of anti-prM pAb with prM protein was inhibited markedly by pr4 in a concentration-dependent manner. c, $\mathbf{d}$ ELISA reactivities of

Our data in this study showed that other four synthetic peptides (pr1, pr3, pr6, and pr9) could not induce high antibody titer in mice. Synthetic peptides representing specific regions of proteins can induce humoral immune responses (Vázquez et al. 2002). It has been reported that short peptide may not exactly imitate the structural of homologous sequence in the native protein (Jemmerson and Hutchinson 1990), because other factors such as neighboring residues and $\mathrm{H}$ bonds (within protein) have also influenced the structure obtained by short stretches in whole protein. Most importantly, peptides must contain potential antigenic sites to elicit B cell interaction.

It has been well demonstrated that the anti-prM antibody mediated DENV infection enhancement and play critical roles
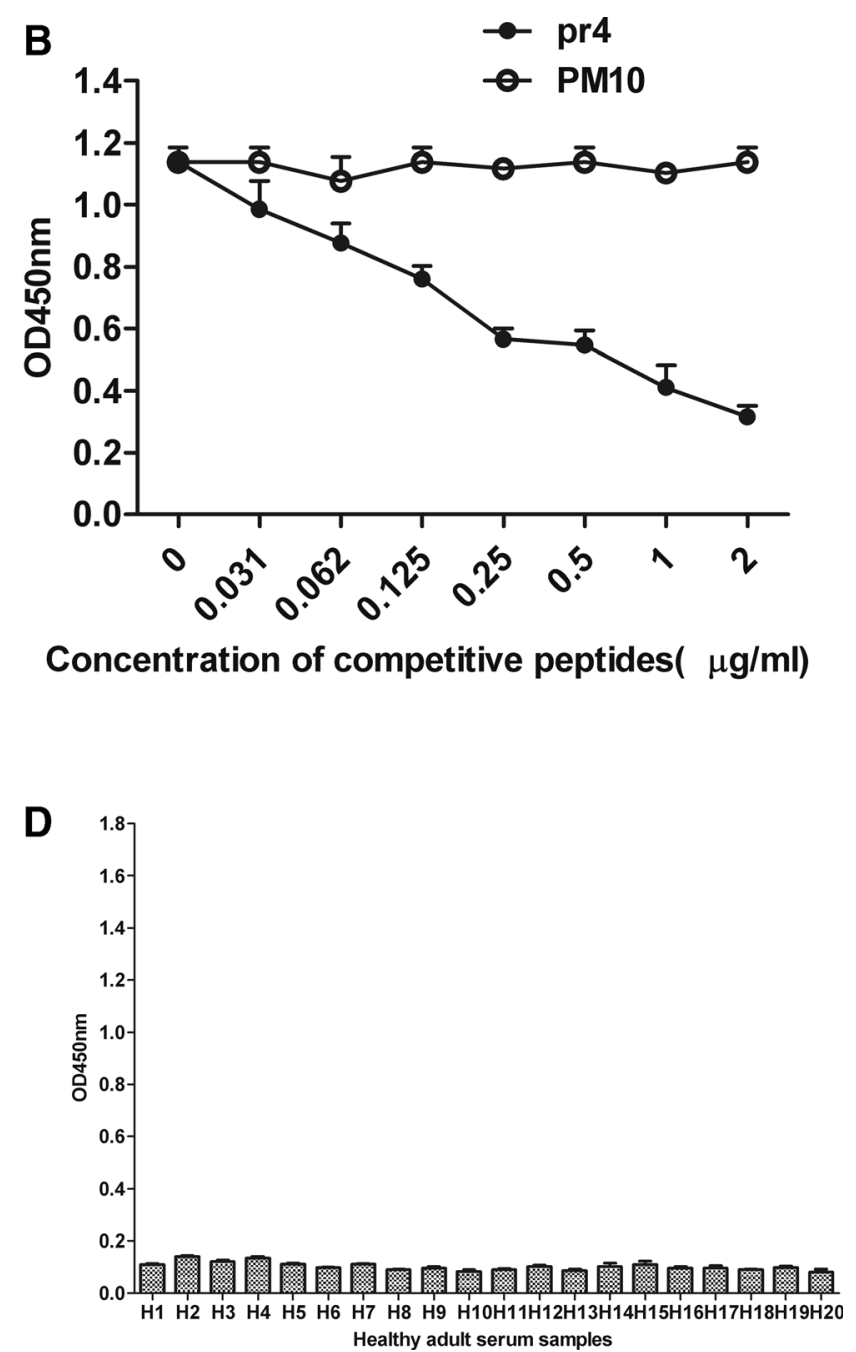

synthetic peptide pr4 with sera from 20 DENV2-infected patients (c) and 20 healthy adults (d). pr4 was able to react with sera from 18 patients infected with DENV2 but not with sera from healthy adults. PM10 was used as control. Data are expressed as means of at least three independent experiments. The error bars represent standard deviations (SD). If there is no error bar, it is not that no variations among three independent experiments but that the variations are too small to show in the figure

in DENV pathogenesis (Rodenhuis-Zybert et al. 2008, 2010; Dejnirattisai et al. 2010; Lai et al. 2008; Beltramello et al. 2010; Huang et al. 2006, 2008; Luo et al. 2013). In agreement with these studies, antibody against epitope peptide pr4 described in this study exhibited broad cross-reactivity and poor neutralizing activity but potent ADE activity toward the four standard DENV serotypes and imDENV. In addition, we found that the epitope peptide pr4 indeed induce enhancing antibodies and lead to an increase of viral load in mice sera during protection assay in vivo. Thus, we concluded that antipr4 serum was infection-enhancing antibody and pr4 was infection-enhancing epitope. Taken together, we further confirmed prM antibodies could enhance infectivity of DENV and demonstrated the importance of prM antibodies during DENV 


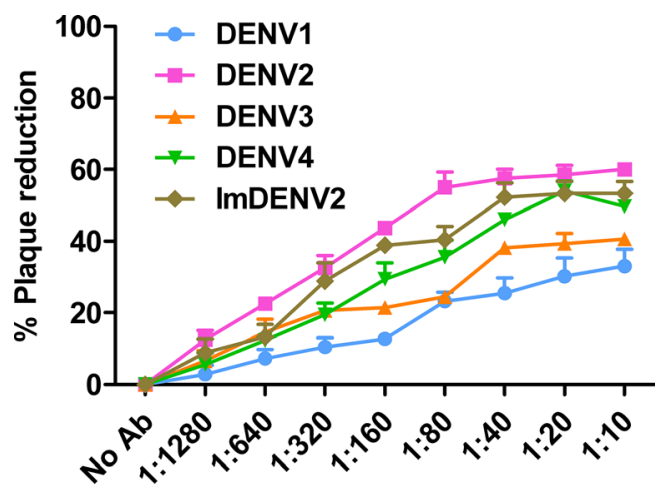

Anti-pr4 sera dilution

Fig. 6 Neutralizing activities of anti-pr4 sera toward standard DENV1-4 and imDENV2. Fifty PFU viruses were mixed with 2-fold serially diluted antibody for $1 \mathrm{~h}$ at $37^{\circ} \mathrm{C}$. Neutralizing activities were detected by plaque reduction assay using BHK-21 cells. Anti-pr4 sera show poor neutralizing activities with standard DENV1-4 and imDENV2. Data are expressed as means of at least three independent experiments. The error bars represent standard deviations (SD). If there is no error bar, it is not that no variations among three independent experiments but that the variations are too small to show in the figure

infection. To date, the molecular mechanism of neutralization and ADE is still not well described. It has been demonstrated that antibodies against prM can enhance infectivity of prMcontaining DENV particles by facilitating efficient binding and cell entry of virus-antibody complexes into Fc receptor-bearing cells (Dejnirattisai et al. 2010; Rodenhuis-Zybert et al. 2010).

Anti-pr4 sera showed different neutralizing and ADE activities against different DENV serotypes, suggesting the structural differences in this region that perhaps involve the polarities and side chain orientations of structurally neighboring amino acids. In addition, the well-exposed epitopes available for antibody recognition in the structural change process are likely to vary in different viruses (Nelson et al. 2008; Roehrig 2003). Also, it has been demonstrated that differences in DENV serotype or even strain might be a factor in generation of varying degrees of $\mathrm{ADE}$ and dengue severity (Endy et al. 2004; Vaughn et al. 2000; Goncalvez et al. 2007). The differences in ADE between serotypes have become the main obstacle to study immunopathogenesis of DENV infection and develop dengue vaccines. Dejnirattisai et al. (2010) and Beltramello et al. (2010) also have found that four DENV serotypes showed different degrees of ADE.

Although dengue vaccine development is considered a global public health priority, approved vaccines remain unavailable due to the cross-reactive among four DENV serotypes and ADE phenomenon. As reported in the present study and recent reports, prM-specific antibodies are capable of restoring and enhancing the infectivity of prM-containing immature and partially mature (Rodenhuis-Zybert et al. 2008, 2010; Dejnirattisai et al. 2010; Lai et al. 2008; Beltramello et al. 2010; Huang et al. 2006, 2008; Luo et al. 2013). Hence, a better vaccine design approach that minimizes the ADE responses elicited by enhancing epitopes in prM protein may contribute to the success of dengue vaccines. Consequently, it may be important to map various kinds of prM epitopes, which are responsible for enhancing antibodies or neutralizing antibodies. However, only a few epitopes in DENV prM protein have been well characterized (Falconar 1999; Huang et al. 2008; Song et al. 2013; Luo et al. 2013). In this study, we map a novel infection-enhancing epitope on dengue pr protein, a finding that may provide new insight into the pathogenesis of DENV infection and contribute to dengue vaccine design.
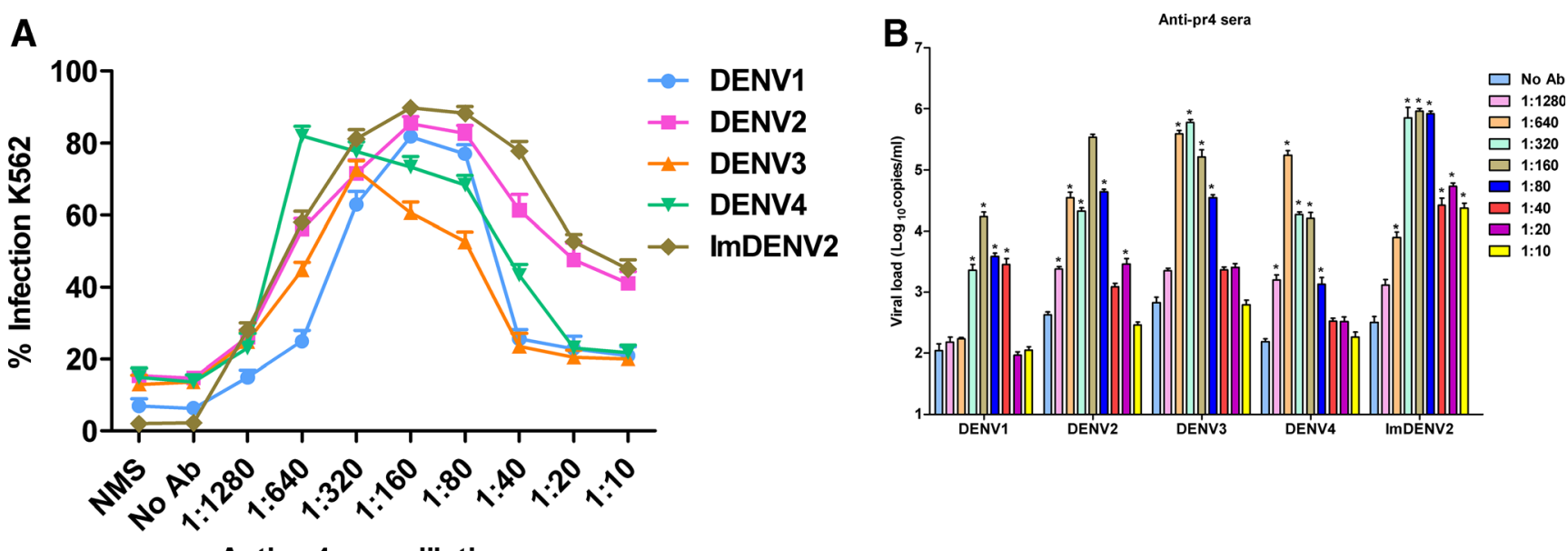

\section{Anti-pr4 sera dilution}

Fig. 7 ADE of DENV infection in K562 cells mediated by anti-pr4 sera. Twofold serially diluted antibodies and an equal volume of DENV (MOI of 1) were mixed for $1 \mathrm{~h}$ at $37^{\circ} \mathrm{C}$ and added to K562 cells. a The percent of infected $\mathrm{K} 562$ cells was measured at 3 days post-infection by flow cytometry. b Viral RNA levels of supernatants were accessed at 4 days post-infection by qRT-PCR. Anti-pr4 sera showed significant ADE activities toward standard DENV and imDENV2. NMS was used as control. Data are expressed as means of at least three independent experiments. The error bars represent standard deviations (SD). If there is no error bar, it is not that no variations among three independent experiments but that the variations are too small to show in the figure. $* p<0.05$ vs No Ab 
In conclusion, we screened a DENV serocomplex crossreactive epitope pr4 (19-34aa) on pr protein using peptide scanning and comprehensive bioinformatics analysis, and found that this epitope was infection-enhancing. These findings may help to understand pathogenesis of DENV infection and advance the development of dengue vaccine.

Acknowledgments We thank Haizhu District Center for Disease Control and Prevention of Guangzhou for providing human serum samples. This study was supported by the Joint National Nature Science Foundation of China and Guangdong Science Foundation Program (U1132002 and U0632002), International (Regional) Joint Research Project (812611 60323), and National Natural Science Foundation of China (31270974).

Conflict of interest There is no conflict of interest.

Ethics statement All procedures involving animals were approved by the Animal Experimentation Ethics Committee of Sun Yat-sen University and carried out by a licensed individual with an ethical approval number of 2012/0081. The use of human sera was approved by the ethical committee of Haizhu District Center for Disease Control and Prevention of Guangzhou, China.

Open Access This article is distributed under the terms of the Creative Commons Attribution License which permits any use, distribution, and reproduction in any medium, provided the original author(s) and the source are credited.

\section{References}

Anandarao R, Swaminathan S, Khanna N (2005) The identification of immunodominant linear epitopes of dengue type 2 virus capsid and NS4a proteins using pin-bound peptides. Virus Res 112:60-68

Balakrishnan T, Bela-Ong DB, Toh YX, Flamand M, Devi S, Koh MB, Hibberd ML, Ooi EE, Low JG, Leo YS, Gu F, Fink K (2011) Dengue virus activates polyreactive, natural IgG B cells after primary and secondary infection. Plos One 6:e29430

Beltramello M, Williams KL, Simmons CP, Macagno A, Simonelli L, Quyen NT, Sukupolvi-Petty S, Navarro-Sanchez E, Young PR, de Silva AM, Rey FA, Varani L, Whitehead SS, Diamond MS, Harris E, Lanzavecchia A, Sallusto F (2010) The human immune response to Dengue virus is dominated by highly cross-reactive antibodies endowed with neutralizing and enhancing activity. Cell Host Microbe 8:271-283

Bhatt S, Gething PW, Brady OJ, Messina JP, Farlow AW, Moyes CL, Drake JM, Brownstein JS, Hoen AG, Sankoh O, Myers MF, George DB, Jaenisch T, Wint GR, Simmons CP, Scott TW, Farrar JJ, Hay SI (2013) The global distribution and burden of dengue. Nature 496: 504-507

Bray M, Lai CJ (1991) Dengue virus premembrane and membrane proteins elicit a protective immune response. Virology 185:505-508

Cardosa MJ, Wang SM, Sum MS, Tio PH (2002) Antibodies against prM protein distinguish between previous infection with dengue and Japanese encephalitis viruses. BMC Microbiol 2:9

Chan AH, Tan HC, Chow AY, Lim AP, Lok SM, Moreland NJ, Vasudevan SG, MacAry PA, Ooi EE, Hanson BJ (2012) A human PrM antibody that recognizes a novel cryptic epitope on dengue $\mathrm{E}$ glycoprotein. PLoS One 7:e33451

Chau TN, Hieu NT, Anders KL, Wolbers M, Lien LB, Hieu LT, Hien TT, Hung NT, Farrar J, Whitehead S, Simmons CP (2009) Dengue virus infections and maternal antibody decay in a prospective birth cohort study of Vietnamese infants. J Infect Dis 200:1893-1900

Cherrier MV, Kaufmann B, Nybakken GE, Lok SM, Warren JT, Chen BR, Nelson CA, Kostyuchenko VA, Holdaway HA, Chipman PR, Kuhn RJ, Diamond MS, Rossmann MG, Fremont DH (2009) Structural basis for the preferential recognition of immature flaviviruses by a fusion-loop antibody. EMBO J 28:3269-3276

Coller BG, Clements DE (2011) Dengue vaccines: progress and challenges. Curr Opin Immunol 23:1-8

Colpitts TM, Rodenhuis-Zybert I, Moesker B, Wang P, Fikrig E, Smit JM (2011) prM-antibody renders immature West Nile virus infectious in vivo. J Gen Virol 92:2281-2285

de Alwis R, Smith SA, Olivarez NP, Messer WB, Huynh JP, Wahala WM, White LJ, Diamond MS, Baric RS, Crowe JE Jr, de Silva AM (2012) Identification of human neutralizing antibodies that bind to complex epitopes on dengue virions. Proc Natl Acad Sci U S A 109: 7439-7444

Dejnirattisai W, Jumnainsong A, Onsirisakul N, Fitton P, Vasanawathana S, Limpitikul W, Puttikhunt C, Edwards C, Duangchinda T, Supasa S, Chawansuntati K, Malasit P, Mongkolsapaya J, Screaton G (2010) Cross-reacting antibodies enhance dengue virus infection in humans. Science 328:745-748

Endy TP, Nisalak A, Chunsuttit wat S, Vaughn DW, Green S, Ennis FA, Rothman AL, Libraty DH (2004) Relationship of preexisting dengue virus (DV) neutralizing antibody levels to viremia and severity of disease in a prospective cohort study of DV infection in Thailand. J Infect Dis 189:990-1000

Falconar AK (1999) Identification of an epitope on the dengue virus membrane $(\mathrm{M})$ protein defined by cross-protective monoclonal antibodies: design of an improved epitope sequence based on common determinants present in both envelope (E and $\mathrm{M})$ proteins. Arch Virol 144:2313-2330

Feng JJ, Luo YY, Zhou JM, Fang DY, Zeng GC, Yan HJ, Jiang LF (2013) Expression of dengue virus type 2 prM protein and analysis of the ADE effect of its polyclonal antibody. $J$ Tropical Med 4:377-381

Goncalvez AP, Engle RE, St Claire M, Purcell RH, Lai CJ (2007) Monoclonal antibody-mediated enhancement of dengue virus infection in vitro and in vivo and strategies for prevention. Proc Natl Acad Sci U S A 104:9422-9427

Gubler DJ (2006) Dengue/dengue haemorrhagic fever: history and current status. Novartis Found Symp 277:3-16

Guy B, Almond J, Lang J (2011) Dengue vaccine prospects: a step forward. Lancet 377:381-382

Guzman MG, Kouri G (2002) Dengue: an update. Lancet Infect Dis 2: $33-42$

Halstead SB (1970) Observations related to pathogenesis of dengue hemorrhagic fever. VI. Hypotheses and discussion. Yale J Biol Med 42: $350-362$

Halstead SB (2003) Neutralization and antibody-dependent enhancement of dengue viruses. Adv Virus Res 60:421-467

Halstead SB, O'Rourke EJ (1977) Antibody-enhanced dengue virus infection in primate leukocytes. Nature 265:739-741

Hua RH, Chen NS, Qin CF, Deng YQ, Ge JY, Wang XJ, Qiao ZJ, Chen WY, Wen ZY, Liu WX, Hu S, Bu ZG (2010) Identification and characterization of a virus-specific continuous B-cell epitope on the PrM/M protein of Japanese Encephalitis Virus: potential application in the detection of antibodies to distinguish Japanese Encephalitis Virus infection from West Nile Virus and Dengue Virus infections. Virol J 7:249

Huang KJ, Yang YC, Lin YS, Liu HS, Yeh TM, Chen SH, Liu CC, Lei HY (2005) Flow cytometric determination for dengue virus-infected cells: its application for antibody-dependent enhancement study. Dengue Bulletin 29:142-150

Huang KJ, Yang YC, Lin YS, Huang JH, Liu HS, Yeh TM, Chen SH, Liu CC, Lei HY (2006) The dual-specific binding of dengue virus and 
target cells for the antibody-dependent enhancement of dengue virus infection. J Immunol 176:2825-2832

Huang KJ, Lin YS, Cheng YT, Huang JH, Liu HS, Yeh TM, Liu CC, Lei HY (2008) Anti-prM antibody as an autoantibody in dengue virus infection. Am J Infect Dis 4:59-67

Hughes HR, Crill WD, Chang GJ (2012) Manipulation of immunodominant dengue virus E protein epitopes reduces potential antibody-dependent enhancement. Virol J 9:115

Jemmerson R, Hutchinson RM (1990) Fine manipulation of antibody affinity for synthetic epitopes by altering peptide structure: antibody binding to looped peptides. Eur J Immunol 20:579-585

Jiang L, Zhou JM, Yin Y, Fang DY, Tang YX, Jiang LF (2010) Selection and identification of B-cell epitope on NS1 protein of dengue virus type 2. Virus Res 150:49-55

Junjhon J, Edwards TJ, Utaipat U, Bowman VD, Holdaway HA, Zhang W, Keelapang P, Puttikhunt C, Perera R, Chipman PR, Kasinrerk W, Malasit P, Kuhn RJ, Sittisombut N (2010) Influence of pr-M cleavage on the heterogeneity of extracellular dengue virus particles. J Virol 84:8353-8358

Lai CY, Tsai WY, Lin SR, Kao CL, Hu HP, King CC, Wu HC, Chang GJ, Wang WK (2008) Antibodies to envelope glycoprotein of dengue virus during the natural course of infection are predominantly crossreactive and recognize epitopes containing highly conserved residues at the fusion loop of domain II. J Virol 82:6631-6643

Li L, Lok SM, Yu IM, Zhang Y, Kuhn RJ, Chen J, Rossmann MG (2008) The flavivirus precursor membrane-envelope protein complex: structure and maturation. Science 319:1830-1834

Li PC, Liao MY, Cheng PC, Liang JJ, Liu IJ, Chiu CY, Lin YL, Chang GJ, Wu HC (2012) Development of a humanized antibody with high therapeutic potential against dengue virus type 2. PLoS Negl Trop Dis 6:e1636

Lin HE, Tsai WY, Liu IJ, Li PC, Liao MY, Tsai JJ, Wu YC, Lai CY, Lu CH, Huang JH, Chang GJ, Wu HC, Wang WK (2012) Analysis of epitopes on dengue virus envelope protein recognized by monoclonal antibodies and polyclonal human sera by a high throughput assay. PLoS Negl Trop Dis 6:e1447

Luo YY, Feng JJ, Fang DY, Jiang LF (2012) Development of TaqMan MGB probe-based real-time fluorescence quantitative reverse transcription PCR for dengue virus and its application. J Mol Diagn Ther 4:158-162

Luo YY, Feng JJ, Zhou JM, Yu ZZ, Fang DY, Yan HJ, Zeng GC, Jiang LF (2013) Identification of a novel infection-enhancing epitope on dengue prM using a dengue cross-reacting monoclonal antibody. BMC Microbiol 13:194

Miller N (2010) Recent progress in dengue vaccine research and development. Curr Opin Mol Ther 12:31-38

Mukhopadhyay S, Kuhn RJ, Rossmann MG (2005) A structural perspective of the flavivirus life cycle. Nat Rev Microbiol 3:13-22

Nelson S, Jost CA, Xu Q, Ess J, Martin JE, Oliphant T, Whitehead SS, Durbin AP, Graham BS, Diamond MS, Pierson TC (2008) Maturation of West Nile virus modulates sensitivity to antibodymediated neutralization. PLoS Pathog 4:e1000060

Rai CI, Lei HY, Lin YS, Liu HS, Chen SH, Chen LC, Yeh TM (2008) Epitope mapping of dengue-virus-enhancing monoclonal-antibody using phage display peptide library. Am J Infect Dis 4:76-84
Rodenhuis-Zybert IA, van der Ende-Metselaar H, Wilschut J, Smit JM (2008) Functional importance of dengue virus maturation: infectious properties of immature virions. J Gen Virol 89:3047-3051

Rodenhuis-Zybert IA, van der Schaar HM, da Silva Voorham JM, van der Ende-Metselaar H, Lei HY, Wilschut J, Smit JM (2010) Immature dengue virus: a veiled pathogen? PLoS Pathog 6: e1000718

Rodenhuis-Zybert IA, Wilschut J, Smit JM (2011) Partial maturation: an immune-evasion strategy of dengue virus? Trends Microbiol 19: $248-254$

Roehrig JT (2003) Antigenic structure of flavivirus proteins. Adv. Virus Res 9:141-175

Se-Thoe SY, Ng MM, Ling AE (1999) Retrospective study of Western blot profiles in immune sera of natural dengue virus infections. $J$ Med Virol 57:322-330

Simmons CP, Chau TN, Thuy TT, Tuan NM, Hoang DM, Thien NT, le Lien B, Quy NT, Hieu NT, Hien TT, McElnea C, Young P, Whitehead S, Hung NT, Farrar J (2007) Maternal antibody and viral factors in the pathogenesis of dengue virus in infants. J Infect Dis 196:416-424

Song KY, Zhao H, Li SH, Li XF, Deng YQ, Wang HJ, Ye Q, Zhu SY, Jiang ZY, Zhang FC, Qin ED, Qin CF (2013) Identification and characterization of a linearized B-cell epitope on the pr protein of dengue virus. Gen Virol 94:1510-1516

Steidel M, Fragnoud R, Guillotte M, Roesch C, Michel S, Meunier T, Paranhos-Baccalà G, Gervasi G, Bedin F (2012) Nonstructural protein NS1 immunodominant epitope detected specifically in dengue virus infected material by a SELDI-TOF/MS based assay. J Med Virol 84:490-499

Vaughn DW, Green S, Kalayanarooj S, Innis BL, Nimmannitya S, Suntayakorn S, Endy TP, Raengsakulrach B, Rothman AL, Ennis FA, Nisalak A (2000) Dengue viremia titer, antibody response pattern, and virus serotype correlate with disease severity. J Infect Dis 181:2-9

Vázquez S, Guzmán MG, Guillen G, Chinea G, Pérez AB, Pupo M, Rodriguez R, Reyes O, Garay HE, Delgado I, García G, Alvarez $M$ (2002) Immune response to synthetic peptides of dengue prM protein. Vaccine 20:1823-1830

Wahala WM, Huang C, Butrapet S, White LJ, de Silva AM (2012) Recombinant dengue type 2 viruses with altered $\mathrm{E}$ protein domain III epitopes are efficiently neutralized by human immune sera. J Virol 86:4019-4023

Wu HC, Huang YL, Chao TT, Jan JT, Huang JL, Chiang HY, King CC, Shaio MF (2001) Identification of B-cell epitope of dengue virus type 1 and its application on diagnosis of patients. J Clin Microbiol 39:977-982

Wu HC, Jung MY, Chiu CY, Chao TT, Lai SC, Jan JT, Shaio MF (2003) Identification of a dengue virus type 2 (DEN-2) serotype-specific Bcell epitope and detection of DEN-2-immunized animal serum samples using an epitope-based peptide antigen. J Gen Virol 84:27712779

Yu IM, Zhang W, Holdaway HA, Li L, Kostyuchenko VA, Chipman PR, Kuhn RJ, Rossmann MG, Chen J (2008) Structure of the immature dengue virus at low $\mathrm{pH}$ primes proteolytic maturation. Science 319: 1834-1837 